\title{
CRIAÇÃO E AVALIAÇÃO DAS QUALIDADES PSICOMÉTRICAS DO QUESTIONÁRIO DE SATISFAÇÃO DOS CLIENTES EM SERVIÇOS DE EDUCAÇÃO FÍSICA (QSCSEF)
}

\author{
MS. MARCELA ALMEIDA ZEQUINÃO \\ Professora de Educação Física, Mestre em Ciências do Movimento Humano \\ - UDESC (Florianópolis - Santa Catarina - Brasil) \\ Email: celaa_@hotmail.com
}

\section{PH.D. FERNANDO LUIZ CARDOSO}

Professor de Educação Física junto ao Programa de Pós-Graduação em Ciências do Movimento Humano (PPGCMH) e do Programa de Pós-Graduação em Educação (PPE);

Coordenador do Laboratório de Gênero, Sexualidade e Corporeidade (LAGESC)

- UDESC (Florianópolis - Santa Catarina - Brasil)

Email: fernandocardoso.ph.d.lagesc@gmail.com

\begin{abstract}
RESUMO
Objetivo: criar e avaliar o Questionário de Satisfação dos Clientes em Serviços de Educação Física (QSCSEF). Método e Resultados: Avaliou-se o construto via avaliação de conteúdo ( $M=9,08)$, clareza $(95,7 \%)$ e análise fatorial (três fatores), bem como a confiabilidade via a técnica das metades partidas $(r=0,772)$ e da consistência interna $(\alpha=0,686)$. Conclusão: - QSCSEF apresentou uma boa reprodutibilidade e consistência interna em termos de confiabilidade. Pode ser considerado claro e válido para mensurar qualidade percebida, expectativa do cliente antes de utilizar o senviço e ocorrência de problemas, como determinantes para satisfação de consumidores de Florianópolis.
\end{abstract}

PALAVRAS-CHAVE: Satisfação dos consumidores; Educação Física e treinamento; questionário; estudos de validação. 
O setor administrativo ainda é uma área pouco explorada pela Educação Física, mas já se apresenta como uma nova e significativa opção para atuação profissional (LOBATO; SILVA, 1998). Apesar de alguns serviços e estabelecimentos que trabalham nesse ramo virem se destacando e melhorando o seu padrão de ação organizacional, são ainda insignificantes diante da excelência a ser alcançada nesse aspecto (LOBATO; VITORINO, 1996). O modelo administrativo dos estabelecimentos esportivos é um exemplo característico da falta de compromisso com a técnica, indicando a carência de uma categoria profissional que possa dar o rumo e a filosofia necessária para que a administração na Educação Física alcance níveis organizacionais mais altos e com maiores perspectivas (LOBATO; VITORINO, 1996, 1997).

Todas as instituições e organizações desportivas devem encarar a administração de forma científica, com base em conhecimentos especializados e como um aspecto fundamental para o alcance dos objetivos propostos, pois se faz necessário entender que o melhor modo para equacionar e solucionar problemas administrativos é fazer uso dos recursos da ciência da Administração (LOBATO; VITORINO, 1997). Estes conhecimentos, aliados à formação técnica em Educação Física e/ ou desportos, torna estes profissionais mais aptos para gerenciar as organizações esportivas, tendo em vista que respeitarão os objetivos e metas do esporte sem desconsiderar os princípios administrativos, assegurando, em longo prazo, o alcance da solidez e a fixação da atividade esportiva como uma meta papável e pertinente (LOBATO; VITORINO, 1996).

A junção do conhecimento em Educação Física com o conhecimento administrativo tornou-se ferramenta fundamental para o sucesso das organizações no segmento esportivo. Com a concorrência neste setor, que tende a oferecer produtos cada vez mais parecidos entre si, e com um consumidor mais consciente e exigente, as empresas obrigam-se a prestar um melhor atendimento, preocupando-se ainda mais com a qualidade e o preço (CONTURSI, 1995; PINHO, 1998), pois todo indivíduo deseja atingir a máxima satisfação pessoal com o menor gasto de tempo, dinheiro e inconvenientes com consequências não planejadas (KOONTZ; O'DONNELL, 1989).

Sendo assim, as instituições esportivas necessitam desenvolver técnicas próprias e adequadas à sua estrutura e, principalmente, possuir avaliação e controle permanentes e efetivos (LOBATO; VITORINO, 1996). A realização deste tipo de procedimento verifica o momento ou a atividade que está sendo desenvolvida, assegurando ao setor administrativo informações e dados para a sua reavaliação e/ou 
redirecionamento (LOBATO; VITORINO, 1997). Entretanto, dentro da Educação Física, a função de avaliação das instituições nem sempre recebe o devido valor e acaba sendo uma das causas dos fracassos neste setor (LOBATO; VITORINO, 1997), pois se sabe que a satisfação dos clientes é um fator crítico para o sucesso, principalmente devido às mudanças ocorridas no cenário econômico mundial, que vêm fazendo com que as organizações e profissionais do treinamento personalizado utilizem estratégias para conseguirem manter-se nesse mercado competitivo (TONTINI; SANT'ANA, 2008; BOSSLE; FRAGA, 20 I I).

O termo satisfação pode ser entendido como a avaliação de uma experiência particular, por isso um constructo abstrato, podendo ser influenciada pelo estado afetivo, gerado pela reação emocional à experiência com um produto ou serviço, que tem relação com o atendimento ou não das necessidades e expectativas do próprio cliente ao longo do tempo (ANDERSON; FORNELL, I994; ANDERSON, FORNELL; LEHMANN, I992; CADOTTE, WOODRUFF; JEKINS, I987; JOHNSON, ANDERSON; FORNELL, 1995; JOHNSON; FORNELL, 1991; OLIVER, I980, 1997; ROSSI; SLONGO, 1998; TINOCO; RIBEIRO, 2007; TONTINI; SANT'ANA, 2008).

Com isso, no setor de prestação de serviços no qual a Educação Física se encaixa, a satisfação dos clientes se torna ainda mais importante, devido à proximidade com o cliente, que exige diálogo ativo entre o mesmo e a empresa (JUNIOR; CARVALHO, 2003). Os serviços podem ser definidos como os resultados gerados por atividades entre o fornecedor e o cliente, bem como pelas atividades internas do fornecedor para atender as necessidades dos mesmos (ASSOCIAÇÃO BRASILEIRA DE NORMAS TÉCNICAS, 1994), ou ainda como um pacote de benefícios implícitos e explícitos, realizados dentro das instalações e utilizando-se de bens facilitadores (FITZSIMMONS; FITZSIMMONS, 2000). O setor de serviços vem crescendo e ganhando importância na economia mundial, reforçando a ideia das empresas estabelecerem estratégias que não só conquistem os consumidores, mas também aproximem e mantenham-nos, conhecendo suas necessidades e expectativas, potencializando os atributos dos serviços prestados, garantindo assim a sobrevivência da organização (SARAVANAN; RAO, 2007; TINOCO; RIBEIRO, 2007).

O nível de satisfação pode ser associado à lealdade dos clientes e consequentemente aos lucros. Níveis elevados de satisfação podem levar à retenção dos consumidores, sendo mais econômico para empresa, pois o custo de manter um cliente antigo é menor que conquistar novos (BATESON; HOFFMAN, 200 I; MILAN; TREZ, 2005; ZACHARIAS, FIGUEIREDO; ALMEIDA, 2008; ZEITHAML, 2003). Além disso, quanto mais tempo o cliente utilizar esse serviço, mais dependente dele se torna, sendo menos suscetível a ofertas de preços menores e ainda 
responsáveis pela propaganda informal, tornando a empresa mais competitiva (MILAN; TREZ, 2005; VAVRA, 1994).

Apesar de estes conceitos serem utilizados universalmente, é importante ressaltar que cada tipo de serviço possui características únicas e específicas que devem ser levadas em consideração quando falamos na medição do nível de satisfação dos clientes (JUNIOR; CARVALHO, 2003). No caso dos serviços relacionados à saúde, como, por exemplo, na Educação Física, mensurar essa satisfação não tem sido fácil, principalmente pela falta de instrumentos científicos padronizados e adequados para esse tipo de atividade (LOPES et al., 2009).

Apesar de saber-se que a adesão a determinadas atividades físicas está altamente relacionada ao grau de prazer e satisfação dos clientes (HENDERSON; AINSWORTH, 2002; WANKEL, 1993; WILLIAMS et al., 2007; WININGER; PARGMAN, 2003), na Educação Física poucas são as organizações que se preocupam em avaliar os seus serviços (MARQUES et al., 20 I I), bem como, os estudos sobre o nível de satisfação dos clientes têm sido escassos, sendo pouco encontrado na literatura pesquisas que visem mensurar como estão sendo prestados os serviços de Educação Física, na perspectiva dos consumidores (CUNNINGHAM, 2007). Com isso, o presente estudo teve como objetivo criar e avaliar o Questionário de Satisfação dos Clientes em Serviços de Educação Física (QSCSEF) em relação à validade e confiabilidade, e apresentará os procedimentos utilizados (teórico, empírico e analítico) e as ferramentas psicométricas adotadas, seguindo os princípios de elaboração de escalas psicométricas de Pasquali (2005), para realizar as análises de conteúdo, clareza, e confiabilidade interna (reprodutividade, Alpha de Cronbach e análise fatorial). Os resultados apresentados a seguir permitirão concluir que QSCSEF apresentou uma boa reprodutibilidade e consistência interna em termos de confiabilidade, e pode ser considerado claro e válido para mensurar qualidade percebida, expectativa do cliente antes de utilizar o serviço e ocorrência de problemas, como determinantes para satisfação de consumidores de Florianópolis.

\section{MÉTODOS}

\section{CRIAÇÃO E TESTAGEM DO QUESTIONÁRIO}

processo de criação e avaliação preliminar do QSCSEF envolveu os seguintes procedimentos: (a) teórico, com a definição do construto e suas dimensões; (b) empírico, com a avaliação do conteúdo e da clareza do instrumento; (c) analítico, com a avaliação da confiabilidade via técnica das metades partidas e Alpha de Cronbach, como também, com a avaliação da validade do construto via análise 
fatorial exploratória. Todos esses recursos foram utilizados para avaliar alguns aspectos da validade e a confiabilidade do QSCSEF. Sendo que uma medida é dita válida se o instrumento de medida consegue mensurar o que se pretende (WIEDERMAN, 2002) e uma medida é dita confiável se é consistente, estável e dependente de sua própria mensuração, o que se refere ao grau de concordância entre múltiplos itens de uma dimensão (confiabilidade interna) e a persistente consistência quando aplicado mais de uma vez com o mesmo indivíduo, por diferentes pesquisadores ou até sob diferentes formas (confiabilidade externa) (ANASTASI, 1986).

\section{PROCEDIMENTOS TEÓRICOS}

Para a elaboração do Questionário de Satisfação dos Clientes com os Serviços de Educação Física, buscou-se uma metodologia que pudesse medir o nível de satisfação dos serviços de forma mais abrangente. Dentre os diversos modelos encontrados, o mais citado e que estava de acordo com os objetivos desse estudo foi o American Customer Satisfaction Index (ACSI) proposto por Fornell et al. ( 1996), traduzido para o português como o Modelo do Índice de Satisfação do Cliente Norte-Americano (ISCN). Esse modelo foi construído e tem sido usado intensamente para analisar sete setores da economia, dentre os quais está presente a prestação de serviços, no ponto de vista dos consumidores (FORNELL et al., 1996; ROSSI; SLONGO, 1998; TINOCO; RIBEIRO, 2007). Segundo os autores do modelo, a satisfação global do cliente não pode ser medida diretamente, sendo necessário mensurar alguns indicadores para obter tal resultado: expectativa do cliente, qualidade percebida e valor percebido. Com base nessas três dimensões, verifica-se a satisfação dos clientes, que resultam na redução ou aumento das reclamações, bem como na lealdade dos mesmos com esses serviços.

Nos estudos feitos, o questionário aplicado consistia em 8 questões demográficas e 17 perguntas, utilizando-se de 15 variáveis para, através de três perguntas, medir a expectativa do cliente, sendo três para a qualidade percebida, duas para o valor percebido, três para a própria satisfação global, uma para reclamações e três para a lealdade. Para mensuração dos dados foi utilizada uma escala de 10 pontos, que permitia uma maior discriminação das respostas por parte dos clientes, bem como auxiliou a redução de problemas estatísticos de distorção nas respostas (FORNELL, 1992). No entanto, apesar de ter-se utilizado no presente estudo o modelo ISCN, após algumas revisões e constatações, embasadas nas sugestões dos avaliadores, responsáveis pela validação de conteúdo (maiores detalhes no tópico "AVALIAÇÃO DE CONTEÚDO"), verificou-se que para alcançar os objetivos propostos, dentro dos serviços de Educação Física, algumas das perguntas do questionário deveriam 
ser retiradas, por acreditar-se que eram redundantes. O Modelo ISCN apresentava duas variáveis observáveis respectivas para valor percebido e três para lealdade, enquanto neste estudo foram utilizadas apenas uma para cada um destes indicadores. Maiores detalhes destas modificações no Quadro 2. Assim, o questionário ficou mais sucinto e objetivo, mesmo sendo um questionário que visa mensurar algo extremamente subjetivo como a satisfação. Dentre outras alterações, temos as adaptações mais específicas à Educação Física, bem como a utilização de uma explicação mais detalhada de cada questão.

A parte inicial do questionário, referente a questões sociais, foi elaborada com o intuito de caracterizar os clientes participantes da pesquisa. Para auxiliar e complementar este questionário acrescentou-se o Critério de Classificação Econômica Brasil - 2008, que tem como objetivo estimar o poder de compra das pessoas e famílias urbanas, não caracterizando a população em classes sociais, mas fazendo uma divisão de classes econômicas (ASSOCIAÇÃO BRASILEIRA DE EMPRESAS DE PESQUISA, 2008).

Outra característica importante desse questionário foi a adaptação da escala de medida, que mensura os dados de 0 à 10, como nos estudos de Tontini e Sant'Ana (2008). Assim conseguem-se os benefícios citados pelo autor do ACSI, sem excluir algo que consideramos importante, que é a total ausência de valor que o zero representa. É importante ressaltar que essa é apenas mais uma escala utilizada para a medição do nível de satisfação, dentre tantas outras existentes, pois não existe uma escala ideal que seja capaz de atender os objetivos de todas as pesquisas de medição de satisfação dos clientes, sendo importante considerar os propósitos de cada uma (ROSSI; SLONGO, 1998).

Assim, o QSCSEF estrutura-se em sete perguntas de ordem social, para caracterização dos participantes, dentre as quais duas fazem parte do Critério de Classificação Econômica Brasil - 2008, para verificar a renda salarial dos mesmos. Em seguida, há uma pergunta sobre quais os serviços de Educação Física que já foram utilizados pelo respondente e por quanto tempo. Por fim, têm-se doze perguntas objetivas, mensuradas em uma escala de 0 à 10 , que visam avaliar o nível de satisfação dos clientes, através de três perguntas referentes à expectativa do cliente com o serviço antes de utilizá-lo, três de qualidade percebida após o uso, uma de valor percebido, três de satisfação, uma de reclamações e uma de lealdade. Têm-se ainda uma pergunta discursiva, onde a intenção é verificar quais os tipos de problemas enfrentados pelos clientes, fazendo dessa pergunta uma variável controle. Maiores detalhes no Apêndice A 


\section{PROCEDIMENTOS EMPÍRICOS}

\section{AVALIAÇÃO DE CONTEÚDO}

A validação de conteúdo do QSCSEF valeu-se da técnica Delphi, que é uma estrutura teórica utilizada que valoriza a participação de indivíduos conhecedores profundos de um determinado assunto e facilita a obtenção de resultados produtivos. É uma técnica não interativa, em que o grupo não se reúne, e que funciona da seguinte forma: $1 .{ }^{\circ}$ - São enviados questionários a cada especialista escolhido, normalmente sobre um cenário; $2^{\circ}$ - Depois de respondidos os questionários são analisados e são resumidas as principais conclusões por parte do pesquisador; 3. - Os questionários voltam aos especialistas, que têm oportunidade de rever as respostas e alterá-las, se for necessário. Se uma resposta varia muito em relação às do resto do grupo, o seu autor terá que justificar a sua diferença de opinião (CUNHA; PENICHE, 2007; FARO, 1997).

Os avaliadores que analisaram o questionário eram doutores pesquisadores. Não foi possível realizar com especialistas na área, devido à dificuldade de encontrar pesquisadores da Educação Física que estudem o tema do presente questionário. Eles utilizaram uma escala para avaliação das questões de 0 a 10 (valor: $0=$ nada válido; de I a 4 = pouco válido; de 5 a 7 = medianamente válido; de 8 a $10=$ válido). Sugestões deveriam ser escritas por extenso abaixo de cada questão. As questões com médias de notas atribuídas abaixo de 7 foram retiradas e as sugestões dos avaliadores consideradas. O prazo estipulado para a devolução das avaliações feitas pelos avaliadores foi de uma semana.

\section{AVALIAÇÃO DE CLAREZA}

Para o teste de clareza, participaram pessoas maiores de 18 anos, que estivessem cursando o Ensino Fundamental, Médio e Superior. Os participantes responderam utilizando uma escala de 0 à 10 (valores: 0 = nada claro; de I a 4 = pouco claro; de 5 a 7 = medianamente claro; e de 8 a $10=$ claro), sendo que as questões que obtivessem médias no valor "medianamente claro" seriam reformuladas e "nada claro" e "pouco claro" excluídas.

\section{PROCEDIMENTOS ANALÍTICOS}

Os procedimentos analíticos são realizados para verificar o grau de confiabilidade e evidências de validade de construto do questionário. Foram efetuados os seguintes procedimentos psicométricos: avaliação da confiabilidade, em termos de metades partidas; medição da confiabilidade interna, através do Alpha de Cronbach; e validade de construto a partir da análise fatorial. 
Através dos procedimentos psicométricos pode-se averiguar a confiabilidade em termos da técnica das metades partidas, que consiste em avaliar a confiabilidade usando respostas obtidas em uma única aplicação do instrumento de medidas (MARTINS, 2006). Nesse sentido, dividiram-se os itens constituintes do questionário em dois grupos: grupo I equivalente as questões "Q.I", "Q.3", "Q.5", "Q.7", "Q.9" e "Q. II" ( $M=45,56 ; S D=7,93)$, e grupo 2 equivalente as questões "Q.2", "Q.4", "Q.6", "Q.8", "Q. I0" e "Q. I2" (M= 45,03; SD = 8,57). A partir desta divisão foi realizada uma correlação para verificar se as duas metades do instrumento estão fortemente relacionadas. Quanto mais semelhantes os escores das duas metades, maior a correlação e mais confiável o instrumento nesse sentido (MARTINS, 2006).

Avaliou-se também a confiabilidade interna por meio do Alpha de Cronbach, verificando a intercorrelação dos itens, e por fim, a validade de constructo através da análise fatorial exploratória, a qual se propõe verificar a dimensionalidade do instrumento através da análise da estrutura fatorial (MARTINS, 2006).

\section{TRATAMENTO DE DADOS}

A proposta de testagem do instrumento necessita de alguns processos em termos de digitação e organização dos dados. Os resultados obtidos nessas diferentes etapas foram tabulados e analisados por estatística descritiva e inferencial no Programa Estatístico SPSS para a versão Windows 20.0. Inicialmente realizou-se o teste de normalidade de Kolmogorov-Smirnov para as doze questões do instrumento, e não se obteve uma distribuição normal. Sendo assim, foram utilizados os seguintes testes estatísticos: Teste de correlação de Spearman para verificar o nível correlação entre as duas metades do QSCSEF, Alpha de Cronbach para avaliar a consistência interna dos itens do questionário, Análise Fatorial Exploratória, pelo Método de Extração Principal Components e Método de Rotação Varimax, para examinar a dimensionalidade do construto elaborado.

\section{PARTICIPANTES}

Após aprovação do Comitê de Ética em Pesquisa da Universidade do Estado de Santa Catarina (processo I |4/2009), foram iniciadas as coletas de dados. Esta pesquisa não probabilística utilizou-se das técnicas snow ball e focus groups para a seleção dos participantes para os três tipos de avaliação: conteúdo, clareza e analítica.

Para a avaliação de conteúdo, participaram 5 doutores pesquisadores do Centro de Ciências da Saúde - CEFID/UDESC. Todos os participantes são coordenadores de laboratórios e possuem funções administrativas tanto dentro do CEFID/UDESC como em outras instituições. Maiores detalhes sobre a formação destes pesquisadores no Quadro I. 


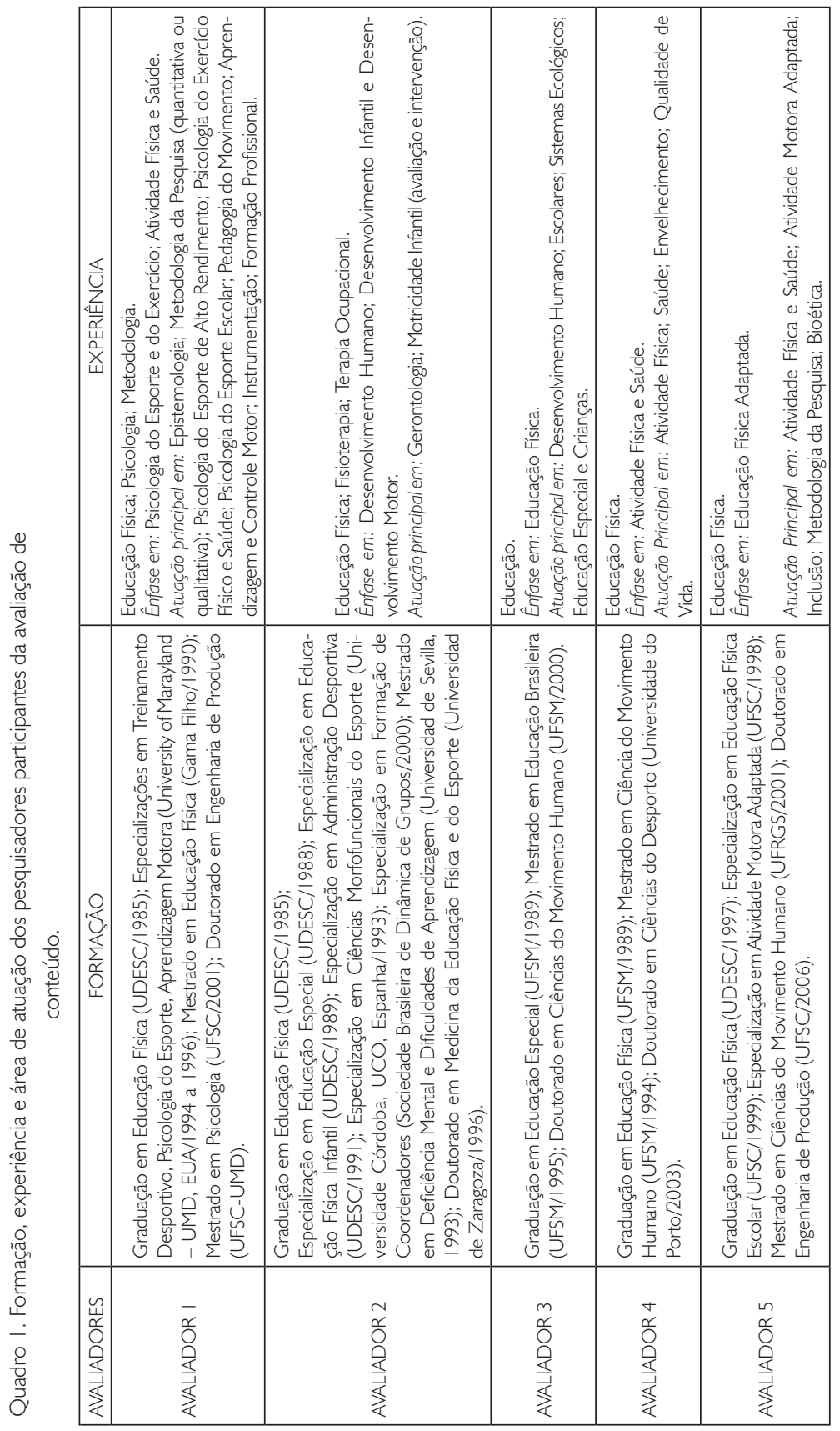


Para avaliação de clareza participaram 167 pessoas dos três níveis de ensino. Para que tivéssemos acesso aos participantes do Ensino Fundamental e Médio, entramos em contato com o Pró-Jovem e com o EJA - SESC, ambos de Florianópolis, dos quais recebemos autorização para aplicar a pesquisa com esses estudantes. Já os do Ensino Superior, foram acadêmicos do curso de Educação Física do CEFID/ UDESC. A idade destes participantes não foi controlada, pois o intuito não era que o instrumento fosse respondido, e sim que fosse dada uma nota de 0 a 10 para o grau de entendimento dos estudantes dos três níveis de ensino em cada questão. Entretanto, todos os participantes desta etapa tinham idade igual ou superior a 18 anos.

Para a validade de constructo participaram 194 pessoas, com a média de idade = 25,21, escolhidas por conveniência, tanto acadêmicos do CEFID/NDESC, bem como em eventos ocorridos em Florianópolis nos meses de setembro e outubro de 2009, como os "Jogos da Diversidade e a Parada da Diversidade".

Com exceção da avaliação de conteúdo, em que as sugestões foram discutidas em particular com cada avaliador, em todas as outras etapas foram mantidas em sigilo a identidade dos participantes. Tanto para as coletas realizadas em salas de aulas, quanto para aquelas realizadas em eventos abertos, os indivíduos recebiam uma cópia do QSCSEF, uma cópia do termo de consentimento e uma caneta para responder o instrumento. Assim que todo o instrumento fosse finalizado, o participante colocava o questionário respondido em uma urna lacrada e o termo de consentimento em outra, para garantir o anonimato.

\section{RESULTADOS}

A análise de conteúdo feita pelos doutores pesquisadores verificou o quanto as perguntas do questionário estavam mensurando o que o instrumento se propunha, ou seja, se estavam de acordo com os objetivos. Todas as perguntas obtiveram médias superiores a 7, não sendo necessárias mudanças significativas. A média de todas as perguntas quanto à sua validade de conteúdo foi $M=9.08$. A menor média apresentada foi $M=7.25$ para a questão "Qual desses serviços você costuma/costumava utilizar para realizar exercícios/atividades físicas e por quanto tempo?", tendo a maior média o valor $M=9.60$, para três questões: "Expectativa de qualidade antes de utilizar o serviço"; "Ocorrência de problemas"; e "Considerando as suas expectativas antes de utilizá-lo, o serviço ficou abaixo ou acima de suas expectativas?".

Apesar de nenhuma questão precisar ser retirada do estudo em função do valor médio obtido na análise dos avaliadores, três questões acabaram sendo retiradas como sugestões dos mesmos, visando tornar o instrumento mais sucinto e objetivo. 
Além disso, foram realizadas algumas alterações na escrita das perguntas e no modo como foram apresentadas, em função das sugestões feitas pelos avaliadores. Estas sugestões foram avaliadas pelos autores e, após análise das mesmas, verificou-se que estas realmente se faziam necessárias e contribuiriam para o aperfeiçoamento do instrumento. Desta forma, em consenso entre os autores, as sugestões foram aceitas e aderidas ao instrumento.

Dentre as principais alterações estão: o acréscimo do instrumento "Critério de Classificação Econômica Brasil - 2008" para a parte sociodemográfica; no item "Q.0" na pergunta sobre qual serviço de Educação Física foi utilizado e por quanto tempo, alterou-se o enunciado, e a opção das alternativas para o tempo de uso foi substituída por uma questão aberta; na I ' Parte - Instruções gerais, foi acrescentado ao enunciado a opção do sujeito escolher sobre qual dos serviços utilizados (assinalado na questão anterior) faria referência às questões respondidas na sequência; em todas as questões do instrumento foram colocadas em destaque as informações trazidas no enunciado referente à descrição dos valores extremos da escala; das duas variáveis observáveis respectivas para valor percebido $(Q .7 ; Q .7 .1)$, uma delas (Q.7.I) foi retirada, bem como das três para lealdade (Q.12; Q.13; Q. 14), duas foram retiradas (Q. I3; Q. I4), sendo assim, neste estudo foram utilizadas apenas uma para cada um destes indicadores; nos itens "Q. I", "Q.2", "Q.4", "Q.5", "Q.7" e "Q.9" foram substituídas algumas palavras das descrições dos valores extremos da escala por outras mais apropriadas, porém mantendo o mesmo significado; no item "Q.6. I" foi colocado antes da pergunta uma instrução indicando se o indivíduo deve responder essa questão ou não; nos itens "Q.7", "Q.8" e "Q. 1 2" foram substituídas algumas palavras do enunciado. Maiores informações no Quadro 2. 


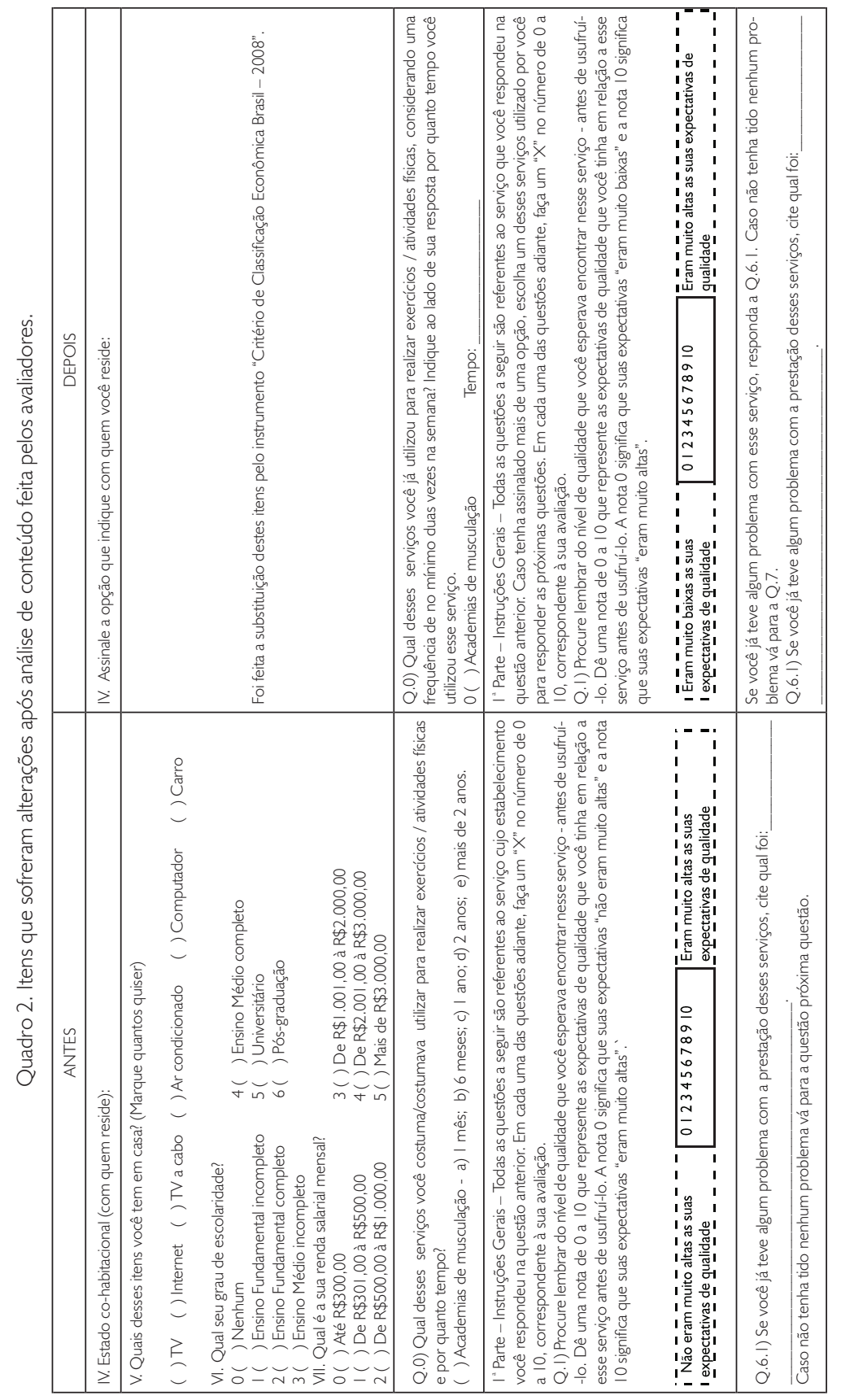




\begin{tabular}{|c|c|c|c|c|c|c|c|}
\hline & 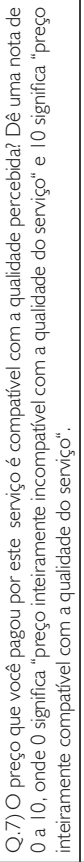 & 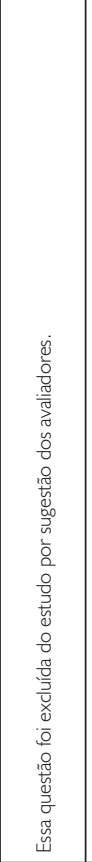 & 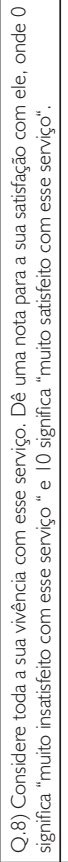 & 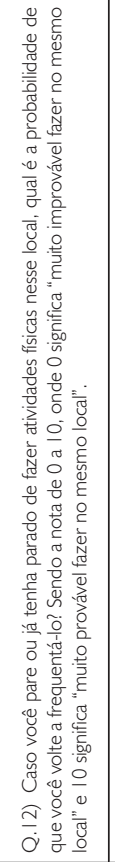 & 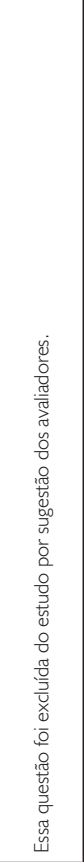 & 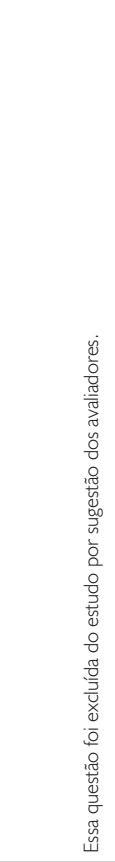 & \\
\hline 岁 & 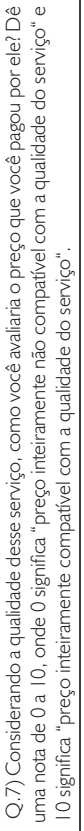 & 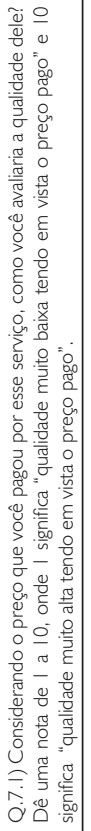 & 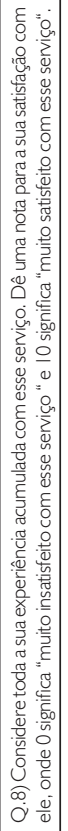 & 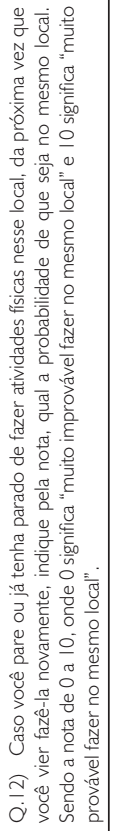 & 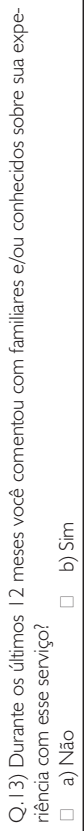 & 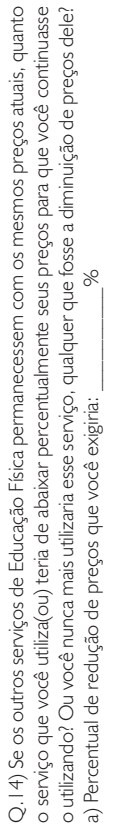 & 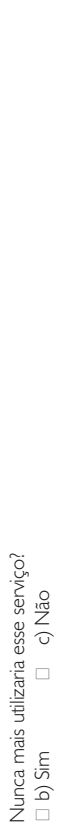 \\
\hline
\end{tabular}


$\mathrm{Na}$ avaliação de clareza, percebeu-se que o QSCSEF apresentou uma pequena variação no seu entendimento, quando aplicado com pessoas de diversos níveis de ensino. O nível de concordância média foi de 95,7\%. Nenhuma pergunta teve um percentual de concordância menor que 70,0\%, o que demonstra um bom entendimento das questões por parte de todos os participantes. As diferenças ficaram entre o grupo do Ensino Fundamental e do Ensino Superior, ficando o Ensino Médio oscilando nos dois extremos. Tem-se, então, que o questionário está mais claro para as pessoas do Ensino Superior e Médio do que para as do Ensino Fundamental, o que não descarta a possibilidade de ser utilizado com esse público, mas esta aplicação em pessoas com apenas ensino básico merece cautela. Maiores detalhes na Tabela I.

Tabela I. Clareza do Questionário de Satisfação dos Clientes com os Serviços de Educação Física.

\begin{tabular}{|c|c|c|c|c|c|c|}
\hline \multirow[t]{3}{*}{ Parâmetros } & \multicolumn{2}{|c|}{$\begin{array}{l}\text { Ensino } \\
\text { Fundamental }\end{array}$} & \multicolumn{2}{|l|}{$\begin{array}{l}\text { Ensino } \\
\text { Médio }\end{array}$} & \multicolumn{2}{|l|}{$\begin{array}{l}\text { Ensino } \\
\text { Superior }\end{array}$} \\
\hline & \multicolumn{2}{|l|}{58} & \multicolumn{2}{|l|}{42} & \multicolumn{2}{|l|}{67} \\
\hline & $\begin{array}{l}\text { \% de con- } \\
\text { cordância }\end{array}$ & & $\begin{array}{l}\text { \% de con- } \\
\text { cordância }\end{array}$ & & $\begin{array}{l}\text { \% de con- } \\
\text { cordância }\end{array}$ & \\
\hline Idade & 94,0 & $\%$ & 97,4 & $\%$ & 99,9 & $\%$ \\
\hline Sexo & 90,2 & $\%$ & 94,3 & $\%$ & 99,1 & $\%$ \\
\hline Orientação sexual & 90,7 & $\%$ & 96,7 & $\%$ & 98,5 & $\%$ \\
\hline Expectativa de qualidade antes de utilizar o serviço & 76,0 & $\%$ & 85,3 & $\%$ & 91,3 & $\%$ \\
\hline Esperava que o serviço atendesse as suas exigências pessoais & 83,1 & $\%$ & 81,7 & $\%$ & 91,4 & $\%$ \\
\hline $\begin{array}{l}\text { Com que frequência esperava ter problemas antes de utilizar } \\
\text { o serviço }\end{array}$ & 81,6 & $\%$ & 85,0 & $\%$ & 92,6 & $\%$ \\
\hline $\begin{array}{l}\text { Avaliação da qualidade do serviço como um todo após } \\
\text { utilizá-lo }\end{array}$ & 82,7 & $\%$ & 85,5 & $\%$ & 93,3 & $\%$ \\
\hline Quão bem o serviço atendeu suas expectativas & 84,1 & $\%$ & 87,6 & $\%$ & 94,2 & $\%$ \\
\hline Ocorrência de problemas & 84,6 & $\%$ & 87,8 & $\%$ & 94,8 & $\%$ \\
\hline Tipos de problemas & 85,5 & $\%$ & 87,1 & $\%$ & 96,0 & $\%$ \\
\hline $\begin{array}{l}\text { Compatibilidade de preço pago com a qualidade do serviço } \\
\text { prestado }\end{array}$ & 83,3 & $\%$ & 90,2 & $\%$ & 95,2 & $\%$ \\
\hline Satisfação com o serviço após utilizá-lo & 85,0 & $\%$ & 89,0 & $\%$ & 93,6 & $\%$ \\
\hline Serviço prestado próximo ou distante do ideal & 85,3 & $\%$ & 91,0 & $\%$ & 94,7 & $\%$ \\
\hline Reclamações do serviço & 86,0 & $\%$ & 89,8 & $\%$ & 96,2 & $\%$ \\
\hline Voltaria a fazer atividades nesse serviço/local? & 83,4 & $\%$ & 92,1 & $\%$ & 95,3 & $\%$ \\
\hline
\end{tabular}

Nota. Os valores referem-se ao percentual de concordância quanto à clareza de cada item atribuídas pelos avaliadores usando uma escala de 0 a 10.

Em relação à confiabilidade em termos de metades partidas "Split-half", realizou-se o teste de correlação de Spearman entre os escores das duas partes, e obteve-se o escore $=0,772$ e $p=0,00 \mathrm{I}$, indicando uma forte correlação entre os dois grupos de questões, sugerindo, então, que o instrumento é confiável. 
Para a análise de confiabilidade interna, considerou-se um $\alpha$ geral $\geq .60$, como sendo moderado em termos de intercorrelação dos itens. Para as doze perguntas do QSCSEF que avaliam satisfação dos alunos/clientes, verificou-se um $\alpha$ geral = 0.686, o que sugere certa consistência e homogeneidade entre as questões (DURIEUX, 2009). O conjunto de itens do fator I, referente à qualidade percebida, apresentou um $\alpha=0,866$; o fator 2 , referente às expectativas antes de utilizar os serviços, apresentou $\alpha=0,724$; e o fator 3, referente a problemas, apresentou um menor $\alpha=0,5$ I5. Mediante a extração de análise dos componentes principais do QSCSEF, observou-se que os dados são apropriados para análise fatorial $(\mathrm{KMO}=0,857$; Barlett $=962,05 ; \mathrm{p}<0.00 \mathrm{I})$.

Além disso, a análise fatorial exploratória também foi realizada, verificando a dimensionalidade do questionário. Assim como no modelo ISCN, escolhido para fundamentar a criação deste questionário, as variáveis carregaram em três dimensões distintas. Através dos resultados, percebemos que as dimensões qualidade percebida e expectativas com os serviços antes de utilizá-lo foram identificadas em nossa análise assim como previa o modelo. Entretanto, surpreendentemente, a terceira dimensão carregada fez referência aos problemas, não diferenciando a pergunta sobre "Ocorrência de problemas" da pergunta "Com que frequência esperava ter problemas antes de utilizar o serviço?", ficando então o valor percebido como sendo parte da dimensão referente à qualidade percebida. A análise indica também que a dimensão qualidade percebida é a responsável pela maior parte do questionário, e consequentemente, da satisfação dos alunos/clientes. Maiores detalhes na Tabela 2.

Tabela 2. Análise Fatorial

\begin{tabular}{|c|c|c|c|}
\hline \multirow[b]{2}{*}{ Parâmetros } & \multicolumn{3}{|c|}{ Dimensões } \\
\hline & $\begin{array}{l}\text { Qualidade } \\
\text { Percebida }\end{array}$ & $\begin{array}{l}\text { Expectativas antes de } \\
\text { utilizar os serviços }\end{array}$ & Problemas \\
\hline Satisfação com o serviço após utilizá-lo & 0,84 & & \\
\hline Serviço ficou abaixo ou acima das expectativas & 0,84 & & \\
\hline Serviço prestado próximo ou distante do ideal & 0,80 & & \\
\hline Quão bem o serviço atendeu as expectativas & 0,80 & & \\
\hline $\begin{array}{l}\text { Avaliação da qualidade do serviço como um todo } \\
\text { após utilizá-lo }\end{array}$ & 0,76 & & \\
\hline Reclamações do serviço & 0,69 & & \\
\hline $\begin{array}{l}\text { Compatibilidade de preço pago com a qualidade do } \\
\text { serviço prestado }\end{array}$ & 0,62 & & \\
\hline Voltaria a fazer atividades nesse serviço/local & 0,60 & & \\
\hline $\begin{array}{l}\text { Esperava que o serviço atendesse as suas exigências } \\
\text { pessoais }\end{array}$ & & 0,87 & \\
\hline Expectativa de qualidade antes de utilizar o serviço & & 0,83 & \\
\hline Ocorrência de problemas & & & 0,80 \\
\hline $\begin{array}{l}\text { Com que frequência esperava ter problemas antes de } \\
\text { utilizar o serviço }\end{array}$ & & & 0,65 \\
\hline Alpha de Cronbach de cada atributo & 0,866 & 0,724 & $0,5 \mid 5$ \\
\hline
\end{tabular}

Nota. Os oito itens do fator "Qualidade percebida" foram responsáveis por 36,33 da variância, já os dois itens do fator "Expectativas antes de utilizar os serviços" foram responsáveis por 13,94 da variância, e por fim os itens do fator "Problemas" foram responsáveis por 13,92 da variância. O total dos três fatores, respondem por 65,20 da variação do instrumento. 


\section{DISCUSSÃO E CONCLUSÃO}

A satisfação de clientes é um tema que vem sendo muito abordado em publicações acadêmicas, sendo uma área em evidência. Sabe-se que os benefícios proporcionados pela pesquisa de satisfação dos clientes são muitos, estando entre eles a melhora do relacionamento da empresa com o cliente; informações mais precisas quanto às necessidades dos clientes; relação de lealdade, com base nas ações corretivas; além de fazer com que os produtores de serviços tenham um índice independente de satisfação do cliente, que os façam apurar suas ofertas, podendo comparar seus resultados com os de outras empresas, e valorizem mais seus consumidores, sendo as informações sobre o nível de satisfação dos clientes uma das prioridades nas organizações comprometidas com a qualidade de seus serviços (MILAN; TREZ, 2005; ROSSI; SLONGO, 1998; URDAN; RODRIGUES, 1999).

Entretanto, na Educação Física ainda são poucos os estudos produzidos na área de marketing e gestão (LOBATO; VALENTE, 1997), principalmente os direcionados à satisfação dos alunos/clientes (CUNNINGHAM, 2007). Sendo assim, não se encontrou nenhum questionário validado no Brasil ou modelo adequado para mensurar essa variável, de forma a nos possibilitar a realização de uma validação concorrente. Além disso, a falta de uma metodologia única para mensurar a satisfação nas outras áreas, principalmente na área da saúde (LOPES et al., 2009), também dificultou a discussão desse artigo e a comparação dos resultados encontrados em relação a outros estudos.

Sabe-se que tornar um conceito subjetivo em algo mensurável não é um procedimento fácil, mas fazem-se necessários mais estudos que visem auxiliar este processo. Por isso, este estudo se propôs, através de ferramentas psicométricas e estatísticas, a ser um ponto de partida, para que mais trabalhos sejam realizados, na tentativa de ampliar os estudos relacionados à Educação Física e à Administração, e que possam ter como base o Questionário de Satisfação dos Clientes com os Serviços de Educação Física.

Para isso, foram adotados os princípios de elaboração de escalas psicométricas de Pasquali (2005), realizando a análise de conteúdo, análise fatorial e clareza, e confiabilidade interna (reprodutibilidade, Alpha de Cronbach). Na validação de conteúdo, o QSCSEF mostrou-se coerente com os aspectos que se propunha avaliar. $\mathrm{Na}$ validação de clareza, este foi considerado claro para ser aplicado sob a forma de questionário, no entanto, uma maior dificuldade em compreender as questões por parte dos menos escolarizados suscita maior cautela nesse processo, mas não 
inviabiliza a sua aplicação. Por exemplo, dar maior ênfase na orientação previa as pessoas menos escolarizadas, ou até mesmo utilizar o QSCSEF como um roteiro de entrevista para pessoas analfabetas ou semianalfabetas.

Sabe-se que o modelo utilizado tem suas qualidades e dificuldades, mas pode ser considerado adequado para medir a satisfação dos clientes em estudos de todo o mundo, podendo atender também as nossas necessidades. Assim como o QSCSEF também apresenta potencialidades e limitações, como por exemplo, os inesperados baixos valores de confiabilidade interna encontrados para o fator denominado "problemas". Contudo, como um passo preliminar em busca de aperfeiçoamento constante, o QSCSEF pode ser considerado uma boa ferramenta de pesquisa na área de satisfação dos clientes de Educação Física.

Sendo assim, pode-se inferir que o Questionário de Satisfação dos Clientes com os Serviços de Educação Física é um instrumento claro, confiável e com conteúdo válido para mensurar as dimensões: qualidade percebida, expectativa do aluno/ cliente antes de utilizar o serviço, e ocorrência de problemas, como determinantes para a satisfação em conformidade com o modelo ISCN utilizado como base para a sua construção (FORNELL et al., 1996). O que o diferencia dos questionários já disponíveis para avaliar a satisfação é que este foi desenvolvido especificamente para os serviços de Educação Física, sendo importante salientar que os resultados encontrados são constatações iniciais, que devem ser reaplicadas em outros ambientes e com outros participantes, para que as conclusões sejam ainda mais fidedignas.

\section{Creation and Evaluation of Psychometric Qualities of the Questionnaire of Customer Satisfaction with the Services in Physical Education (QCSSPE)}

ABSTRACT: Objective: To build and evaluate the Questionnaire of Customer Satisfaction with the Services in Physical Education (QCSSPE). Method and Results: We evaluated the instrument construct using content rating $(M=9,08)$, clarity $(95,7 \%)$ and the factorial analyses (three factors). Besides that, we tested the reliability using the split half technique $(r=0,772)$ and the internal consistence $(\alpha=0,686)$. Conclusion: QCSSPE showed good reproducibility and internal consistency in terms of reliability and can be considered clear and valid to measure perceived quality, customer expectations before using the service and the occurrence of problems such as determinants of consumer satisfaction in Florianópolis.

KEYWORDS: Customer satisfaction; Physical Education and training; questionnaire; validation studies. 


\section{Creación y evaluación de las cualidades psicométricas del Cuestionario de Satisfacción del Cliente acerca de los Servicios en Educación Física (CSCSEF)}

RESUMEN: Objetivo: construir y evaluar el Cuestionario de satisfacción del cliente con los servicios en Educación Física (QSCSEF). Método y Resultados: el constructo fue evaluado a partir del contenido $(M=9,08)$, claridad $(95,7 \%)$ y análisis factorial (tres factores); la confiabilidad fue obtenida con el método de las dos mitades $(r=0,772)$ y análisis de la consistencia interna $(\alpha=0,686)$. Conclusión: el QSCSEF presentó una buena reproductibilidad y consistencia interna. Puede ser considerado claro y válido para medir la calidad percibida, las expectativas del cliente antes de usar el servicio y la aparición de problemas tales como factores determinantes de la satisfacción de los consumidores en Florianópolis.

PALABRAS CLAVE: Satisfacción de los consumidores; educación y entrenamiento físico; cuestionario; estudios de validación.

\section{REFERÊNCIAS}

ANASTASI, A. Evolving concepts of test validation. Annual Review Psychology, v. 37, p, I- I 5, 1986.

ANDERSON, E. FORNELL, C. Customer satisfaction research prospectus. In: RUST, R; OLIVER, R. Service quality: new directions in theory and practice. Thousand Oaks: Sage, 1994.

ANDERSON, E. FORNELL, C.; LEHMANN, D. Perceived quality, customer satisfaction, market share, and profitability. Michigan: University of Michigan, 1992.

ASSOCIAÇÃO BRASILEIRA DE EMPRESAS DE PESQUISA. Critérios de classificação econômica Brasil, 2009.

ASSOCIAÇÃO BRASILEIRA DE NORMAS TÉCNICAS (ABNT). NBR ISO 8402: 1 994: Gestão da qualidade e garantia da qualidade - Terminologia. Rio de Janeiro: ABNT, 1994.

BATESON, J., HOFFMAN, K. Marketing de serviços. Porto Alegre: Bookman, $200 \mathrm{I}$.

BOSSLE, C. B; FRAGA, L. B. O personal trainer na perspectiva do marketing. Revista Brasileira de Ciências do Esporte, v. 33, n. I, 201 I.

CADOTTE, E., WOODRUFF, R; JENKINS, R. Expectations and norms in models of consumer satisfaction. Journal of Marketing Research, v. 24, p. 305-314, 1987.

CONTURSI, E. (1995). Criatividade. Sprint Magazine (76).

CORRER, C. et al. Satisfação dos usuários com serviços da farmácia: Tradução e validação do Pharmacy Services Questionnaire para o Brasil. Cadernos de Saúde Pública,v. 25, n. I, p. 87-96, 2009. 
CUNHA, A., PENICHE, A. Validação de um instrumento de registro para sala de recuperação pós anestésica. Acta Paulista de Enfermagem,v. 20, n. 2, p. I51-160, 2007.

CUNNINGHAM, G. Development of the physical activity class satisfaction questionnaire. Measurement in Physical Education and Exercise Science, v. II , n.3, p. 161-176, 2007.

DURIEUX, A. Validação do 'Cade Q': Questionário para avaliar o conhecimento sobre a doença arterial coronariana em pacientes com DAC em programas de reabilitação cardiopulmonar e metabólica.2009-(Mestrado), Universidade do Estado de Santa Catarina, Florianópolis, 2009.

FAIRCLOUGH, D.; GELBER, R. Quality of life: statistical issues and analysis. In B. SPILKER (Ed.), Quality of life and pharmacoeconomics in clinical trials. New York: Lippincott Williams \& Wilkins,v. 2, 1996.

FARO, A. Técnica Delphi na validação das intervenções de enfermagem. Revista da Escola de Enfermagem, São Paulo, v. 33, p. 279-283, 1997.

FITZSIMMONS, J.; FITZSIMMONS, M. Administração de serviços: operações, estratégia e tecnologia de informação. Porto Alegre: Bookman, 2000.

FORNELL, C. A national customer satisfaction barometer: the swedish experience. Journal of Marketing, n. 56, p. 6-21, 1992.

FORNELL, C. et al. The american customer satisfaction index: nature, purpose, and findings. Journal of Marketing,v. 60, n. 4, p. 7 - 12, 1996.

HENDERSON, K.; AINSWORTH, B. Enjoyment: a link to physical activity, leisure, and health. Journal of Park and Recreation Administration,v.20, n.4, p. 130-146, 2002.

JOHNSON, M; ANDERSON, E.; FORNELL, C. Rational and adaptive performance expectations in customer satisfaction framework. Journal of Consumer Research, v. 21, p. 695-707, 1995.

JOHNSON, M.; FORNELL, C. (1991). A framework for comparing customer satisfaction across individuals and product categories. Journal of Economic Psychology, v. 12, n. 2, p. 267-286, 1991.

JUNIOR, A.; CARVALHO, M. Obtenção da voz do consumidor: estudo de caso em um hotel ecológico. Produção, v. 13, n.3, p. 88-100, 2003.

KOONTZ, H.; O’DONNELL, C. Fundamentos à administração. São Paulo: Pioneira, v. 2, 1989.

LOBATO, P.; SILVA, S. Gestão organizada e Educação Física: um caminho para a excelência. Revista Mineira de Educação Física, Viçosa, v. 6, n. I, p. 18-28, 1998.

LOBATO, P.; VALENTE, M. ( 1997). O papel da comunicação no marketing esportivo. Revista Mineira de Educação Física, Viçosa, v. 5, n. I, p. 40-55, 1997. 
LOBATO, P.; VITORINO, E. O papel da administração esportiva no desenvolvimento do esporte universitário. Revista Mineira de Educação Física, Viçosa, v. 4, p. 6I-64, 1996.

LOBATO, P.; VITORINO, E. O perfil administrativo adequado a uma instituição desportiva em relação aos princípios teóricos da Administração.Motriz ,v. 3, n. 2, p. 95-103, 1997.

LOPES, J. et al. Satisfação de clientes sobre cuidados de enfermagem no contexto hospitalar. Acta Paulista de Enfermagem, São Paulo, v. 22, n. 2, p. I36-141, 2009.

MARQUES, A. I. et al. Evaluation of physical activity programmes for the elderly - exploring the lessons from other sectors and examining the general characteristics of the programmes. BMC Res Notes,v. 4, n. I, p. 368, 201 I.

MARTINS, G. D. A. Sobre confiabilidade e validade. Revista Brasileira de Gestão de Negócios,v. 8, n. 20, p. I-12, 2006.

MILAN, G.; TREZ, G. Pesquisa de satisfação: um modelo para planos de saúde. RAE eletrônica,v. 4, n.2, p. I-2I, 2005.

OLIVER, R. A cognitive model of the antecedents and consequences of satisfaction decisions. Journal of Marketing Research, v. 17, p. 460-469, 1980.

OLIVER, R. Satisfaction: a behavioral perspective on the consumer. New York: Irwin/McGrawHill, 1997.

PASQUALI, L. Elaboração de instrumentos psicológicos. São Paulo: Casa do psicólogo, 2005. PINHO, J. Comunicação em Marketing Esportivo. São Paulo: Summus, 1998.

ROSSI, C.; SLONGO, L. Pesquisa de satisfação de clientes: o estado-da-arte e proposição de um método brasileiro. Revista de Administração Contemporânea, v. 2, n. I, p. 10 l - 125, 1998.

SARAVANAN, R.; RAO, K. Service quality from the customer's perspective: an empirical investigation. Quality Management Journal, v. I4, n. 3, p. I5-24, 2007.

TINOCO, M.; RIBEIRO, J. Uma nova abordagem para a modelagem das relações entre os determinantes da satisfação dos clientes de serviços. Produção, v. 7, n. 3, p. 454-470, 2007.

TONTINI, G.; SANT'ANA, A. Interação de atributos atrativos e obrigatórios de um serviço na satisfação do cliente. Produção, v. 18, n. I, p. I I2-125, 2008.

URDAN, A.; RODRIGUES, A. O modelo do índice de satisfação do cliente norte-americano: um exame inicial no Brasil com equações estruturais. Revista de Administração Contemporânea - RAC, v. 3, n. 3, p. 109-130, 1999.

VAVRA, T. Selling after the sale: the advantages of aftermarketing. Supervision, v. 50, n. 10 , p. 9-12, 1994. 
WANKEL, L. The importance of enjoyment to adherence and psychological benefits from physical activity. Journal of Sport Psychology, v. 24, p. 151-169, 1993.

WILLIAMS, N. H. et al. Effectiveness of exercise-referral schemes to promote physical activity in adults: systematic review. BrJ Gen Pract, v. 57, n. 545, p. 979-986, 2007.

WININGER, S.; PARGMAN, D. Assessment of factors associated with exercise enjoyment. Journal of Music Therapy, v. 40, n. I, p. 57-73, 2003.

ZACHARIAS, M., FIGUEIREDO, K.,; ALMEIDA, V. Determinantes da satisfação dos clientes com serviços bancários. Revista de Admnistração de Empresas Eletrônica, v.7, n.2, 2008.

ZEITHAML, V. Marketing de serviços: a empresa com foco no cliente. Porto Alegre: Bookman, 2003.

WIEDERMAN, M. Reliability and validity of measurement. In WIEDERMAN,M; WHITLEY,B. Whitley (Ed.) Handbook for conducting research on human sexuality. Mahwah: Lawrence Erlbaum, 2002.

Recebido em: 27 set. 2011 Aprovado em: 17 abr. 2012

Endereço para Correspondência: Fernando Luiz Cardoso, Ph.D. Rua Arno Hoeschl, I59, apto 1200.

Centro / Florianópolis / SC / Brasil CEP 88.0। 5-620 